\title{
Article \\ Convergence on Population Dynamics and High-Dimensional Haddock Conjecture
}

\author{
Wenke Wang ${ }^{1}{ }^{1}$, Le $\mathrm{Li}^{1}$, Xuejun $\mathrm{Yi}^{2}$ and Chuangxia Huang ${ }^{3, *} \mathbb{D}$ \\ 1 College of Meteorology and Oceanography, National University of Defense Technology, \\ Changsha 410073, China; wangwenke@nudt.edu.cn (W.W.); lile8621@hnu.edu.cn (L.L.) \\ 2 School of Mathematics, Hunan University, Changsha 410082, China; s2xuejun@hnu.edu.cn \\ 3 Hunan Provincial Key Laboratory of Mathematical Modeling and Analysis in Engineering, \\ Changsha University of Science and Technology, Changsha 410114, China \\ * Correspondence: cxiahuang@csust.edu.cn
}

check for updates

Citation: Wang, W.; Li, L.; Yi, X.; Huang, C, Convergence on

Population Dynamics and High Dimensional Haddock Conjecture. Symmetry 2021, 13, 2252. https:// doi.org/10.3390/sym13122252

Academic Editor: Eulalia Martínez Molada

Received: 10 October 2021

Accepted: 18 November 2021

Published: 26 November 2021

Publisher's Note: MDPI stays neutral with regard to jurisdictional claims in published maps and institutional affiliations.

Copyright: (C) 2021 by the authors. Licensee MDPI, Basel, Switzerland. This article is an open access article distributed under the terms and conditions of the Creative Commons Attribution (CC BY) license (https:/ / creativecommons.org/licenses/by/ $4.0 /)$.

\begin{abstract}
One fundamental step towards grasping the global dynamic structure of a population system involves characterizing the convergence behavior (specifically, how to characterize the convergence behavior). This paper focuses on the neutral functional differential equations arising from population dynamics. With the help of monotonicity techniques and functional methods, we analyze the subtle relations of both the $\omega$-limited set and special point. Meanwhile, we prove that every bounded solution converges to a constant vector, as $t$ tends to positive infinity. Our results correlate with the findings from earlier publications, and our proof yields an improved Haddock conjecture.
\end{abstract}

Keywords: convergence; population dynamics; neutral functional differential equation; Haddock conjecture

\section{Introduction}

It is well known that a delay differential equation model plays an important role in the process of population dynamics modeling, especially when the species clearly goes through the incubation period and evolves at different stages [1-4]. In addition to stability, limited cycles, bifurcation, and periodic solutions-how to characterize convergence behavior is a fundamental and important step toward grasping the global dynamic structure of a population system [5-12]. As early as 1976, at an international conference, Bernfeld and Haddock noted that every solution of the scalar population delay differential equation converges to a constant [13]

$$
[x(t)-p x(t-r)]^{\prime}=-x^{\frac{1}{3}}(t)+x^{\frac{1}{3}}(t-r), \quad r>0 .
$$

Consequently, the above scalar neutral functional differential equation (NFDE) has been naturally generalized to the following autonomous NFDE [14]

$$
[x(t)-p x(t-r)]^{\prime}=-F(x(t))+G(x(t-r))
$$

and non-autonomous NFDE [15]

$$
[x(t)-p x(t-\sigma(t))]^{\prime}=b(t)[-F(x(t))+F(x(t-\sigma(t)))],
$$

respectively. Here, $F \in C\left(\mathbb{R}^{1}, \mathbb{R}^{1}\right)$ is strictly increasing, $G \in C\left(\mathbb{R}^{1}, \mathbb{R}^{1}\right)$, and $\sigma, b \in C\left(\mathbb{R}^{1}, \mathbb{R}^{1}\right)$ are bounded. 
In fact, several important infinite dimensional dynamical systems arising from population growth are special cases of the following model $[5,16,17]$

$$
\left\{\begin{array}{rlll}
\left(x_{1}(t)-c_{2} x_{2}\left(t-r_{2}\right)\right)^{\prime} & = & -F\left(x_{1}(t)\right)+G\left(x_{2}\left(t-r_{2}\right)\right), \\
\left(x_{2}(t)-c_{3} x_{3}\left(t-r_{3}\right)\right)^{\prime} & = & -F\left(x_{2}(t)\right)+G\left(x_{3}\left(t-r_{3}\right)\right), \\
& \ldots & \ldots & \\
\left(x_{n-1}(t)-c_{n} x_{n}\left(t-r_{n}\right)\right)^{\prime} & = & -F\left(x_{n-1}(t)\right)+G\left(x_{n}\left(t-r_{n}\right)\right), \\
\left(x_{n}(t)-c_{1} x_{1}\left(t-r_{1}\right)\right)^{\prime} & = & -F\left(x_{n}(t)\right)+G\left(x_{1}\left(t-r_{1}\right)\right),
\end{array}\right.
$$

where $F$ describes the instantaneous mortality rate, and $G \in C\left(\mathbb{R}^{1}, \mathbb{R}^{1}\right)$ is the feedback function incorporating with positive delays $r_{1}, \cdots, r_{n}$. Clearly, system (1) can be considered as a neutral delayed cellular neural network accompanying $D$ operators [18]. When $F$ is a strictly monotonically increasing function, $c_{i} \in(0,1), r_{i}>0$ for $i \in \Omega=\{1,2, \cdots, n\}$, and either $G(x) \leq F(x)$ or $G(x) \geq F(x)$ for all $x$, systems (1) has been used to confirm the high-dimensional Haddock conjecture $[13,15,16]$.

Although the scalar Haddock conjecture has been successfully demonstrated by the authors in the literature $[14,15]$, the high-dimensional structure brings practical difficulties to prove the high-dimensional Haddock conjecture based on the system (1). This makes the existing proofs of the high-dimensional Haddock conjecture to be done only with with additional conditions. In particular, when $n=2$, for the system (1), the authors in [16] proved that each solution converges to a constant vector, as long as the solution is bounded. Furthermore, the authors in [17] noted that it is necessary to add one of the following assumptions to ensure the correctness of the main results in [13].

- $\quad\left(\mathbf{S}_{+}\right)$Assume $G \geq F$, and if $I$ is an any given bounded interval on $\mathbb{R}^{1}$, then there is a constant $\mu>0$ such that $F\left(x_{2}\right)-F\left(x_{1}\right) \leq \mu\left(x_{2}-x_{1}\right)$ for any $\left[x_{1}, x_{2}\right] \subseteq I$.

- $\quad$ (S-) Assume $G \leq F$, and if $I$ is an any given bounded interval on $\mathbb{R}^{1}$, then there is a constant $\mu^{\prime}>0$ such that $F\left(x_{2}\right)-F\left(x_{1}\right) \geq \mu^{\prime}\left(x_{2}-x_{1}\right)$ for any $\left[x_{1}, x_{2}\right] \subseteq I$.

Later on, under the technical assumptions, two-dimensional cases of systems (1) are investigated in [19-22] as well. Regrettably, if one chooses $G(x) \equiv F(x)=x^{\frac{1}{3}}$ designed in the Haddock conjecture, simple verification can guide that the function $F$ and $G$ do not meet the requirements of conditions $\left(\mathbf{S}_{+}\right)$or $\left(\mathbf{S}_{-}\right)$. Therefore, even involving the assumptions $\left(\mathbf{S}_{+}\right)$and $\left(\mathbf{S}_{-}\right)$, for high-dimensional neutral functional differential equations (1), the Haddock conjecture is still untenable. This motivates us to remove the assumptions $\left(\mathbf{S}_{+}\right)$ and $\left(\mathbf{S}_{-}\right)$, and generalize the Haddock conjecture to a high-dimensional version (1) to reveal that every bounded solution converges to a constant.

Without adopting the above-mentioned assumptions $\left(\mathbf{S}_{+}\right)$and $\left(\mathbf{S}_{-}\right)$, in the following section, we develop the delicate monotonicity with systems (1) and analyze the subtle relation of both the $\omega$-limited set and special point with the help of monotonicity techniques and functional methods. In Section 3, the main results are presented and demonstrated by applying the preliminary lemmas of Section 2. Moreover, our results in this paper correlate with earlier publications, and Corollary 1 yields an improved Haddock conjecture.

\section{Preliminaries}

For convenience, we present the following notations. We denote

$$
\begin{aligned}
& C=\prod_{i=1}^{n} C\left(\left[-r_{i}, 0\right], \mathbb{R}^{1}\right), \quad|r|=\sum_{i=1}^{n} r_{i}, \quad \Omega=\{1,2, \cdots, n\}, \\
& \tau=\min _{j \in \Omega} r_{j}, \quad \bar{j}=j \text { for } j \in \Omega, \quad \overline{0}=n, \overline{n+1}=1 .
\end{aligned}
$$

Equipping with the usual supremum norm, it is easy to see that $C$ is a Banach space. We also define the order cones $K$ in the following way:

$$
K=\left\{\varphi \in C_{+}: \varphi_{i}(0)-c_{\overline{i+1}} \varphi_{\overline{i+1}}\left(-r_{\overline{i+1}}\right) \geq 0 \text { for } i \in \Omega\right\},
$$


where

$$
C_{+}=\prod_{i=1}^{n} C\left(\left[-r_{i}, 0\right], \mathbb{R}_{+}^{1}\right) .
$$

For $A \subseteq C$, and $\forall \phi_{1}, \phi_{2} \in C$, we designate

- $\phi_{1} \ll \phi_{2}$ iff $\phi_{2}-\phi_{1} \in \operatorname{Int} C_{+} ; \quad \phi_{1} \ll_{K} \phi_{2}$ iff $\phi_{2}-\phi_{1} \in \operatorname{Int} K ; \quad \phi_{1} \ll_{K} A$ iff $\phi_{1} \ll_{K} \phi_{2}$ for all $\phi_{2} \in A$.

- $\phi_{1} \leq \phi_{2}$ iff $\phi_{2}-\phi_{1} \in C_{+} ; \quad \phi_{1} \leq_{K} \phi_{2}$ iff $\phi_{2}-\phi_{1} \in K ; \quad \phi_{1} \leq_{K} A$ iff $\phi_{1} \leq_{K} \phi_{2}$ for all $\phi_{2} \in A$.

- $\quad \phi_{1}<\phi_{2}$ iff $\phi_{1} \leq \phi_{2}$ and $\phi_{1} \neq \phi_{2} ; \quad \phi_{1}<_{K} \phi_{2}$ iff $\phi_{1} \leq_{K} \phi_{2}$ and $\phi_{1} \neq \phi_{2} ; \quad \phi_{1}<_{K} A$ iff $\phi_{1}<_{K} \phi_{2}$ for all $\phi_{2} \in A$.

It is worth noting that $\phi_{1} \gg_{K} \phi_{2}$ and $\phi_{1} \geq_{K} \phi_{2}$ can be defined naturally. For $i \in \Omega, \sigma>0, x_{i} \in C\left(\left[-r_{i}, \sigma\right], \mathbb{R}^{1}\right)$, it is obvious that $x_{t} \in C$ can be presented as $x_{t}=\left(x_{1, t}, x_{2, t}, \cdots, x_{n, t}\right)$ and $x_{i, t}(\xi)=x_{i}(t+\xi)$ for all $\xi \in\left[-r_{i}, 0\right], t \in[0, \sigma]$. Furthermore, we also define

$$
\hat{\beta}=\left((\widehat{\beta})_{1},(\widehat{\beta})_{2}, \cdots,(\widehat{\beta})_{n}\right),
$$

in which $(\widehat{\beta})_{i}(\xi)=\beta, i \in \Omega, \xi \in\left[-r_{i}, 0\right]$. For the sake of simplicity, if $\varphi \in C$ is an initial value, we use the notation $x_{t}(\varphi)(x(t, \varphi))$ to represent the solution of the initial value problem of systems (1).

Remark 1. Assume there are $\alpha \in \mathbb{R}^{1}$ and $\varphi \in C$, such that $\varphi \geq_{K} \widehat{\alpha}$, then it is easy to establish two statements as below:

- $\quad$ (i) If $\varphi_{i}(0)-c_{\overline{i+1}} \varphi_{\overline{i+1}}\left(-r_{\overline{i+1}}\right)>\left(1-c_{\overline{i+1}}\right) \alpha$ for some $i \in \Omega$, then $\varphi_{i}(0)>\alpha$.

- (ii) If $\varphi_{i}(0)-c_{\overline{i+1}} \varphi_{\overline{i+1}}\left(-r_{\overline{i+1}}\right)=\left(1-c_{\overline{i+1}}\right) \alpha$ for some $i \in \Omega$, then either $\varphi_{i}(0)=\varphi_{\overline{i+1}}\left(-r_{\overline{i+1}}\right)=\alpha$ or $\varphi_{i}(0)<\varphi_{\overline{i+1}}\left(-r_{\overline{i+1}}\right)$.

Remark 2. Assume that $\sigma>0, x_{i} \in C\left(\left[-r_{i}, \sigma\right], \mathbb{R}^{1}\right)$ for $i \in \Omega$ and $x_{0} \geq_{K} \widehat{\alpha}$, it is easy to obtain the following statements:

- (i) For all $t \in[0, \sigma]$ and $i \in \Omega$, if $x_{i}(t)-c_{\overline{i+1}} x_{\overline{i+1}}\left(t-r_{\overline{i+1}}\right) \geq\left(1-c_{\overline{i+1}}\right) \alpha$, then, for all $t \in[0, \sigma]$, we have $x_{t} \geq_{K} \widehat{\alpha}$.

- (ii) If $\sigma \geq \max _{i \in \Omega} r_{i}$, and $x_{t} \geq_{K} \widehat{\alpha}$ holds for any $t \in[0, \sigma]$, furthermore, $x_{i}(t)-c_{\overline{i+1}} x_{\overline{i+1}}$ $\left(t-r_{i+1}\right)>\left(1-c_{i+1}\right) \alpha$ holds for all $t \in\left[\sigma-r_{i}, \sigma\right]$ and $i \in \Omega$, then we have $x_{\sigma} \gg_{K} \widehat{\alpha}$.

In this section, for all $x \in \mathbb{R}^{1}$, we always assume that the inequality $G(x) \geq F(x)$ holds. Hereafter, we denote $\sum_{j=n+1}^{n} r_{j}=0, \sum_{j=n+2}^{n} r_{j}=-r_{1}$, and suppose that $F$ is a strictly increasing function defined on $\mathbb{R}^{1}$.

Lemma 1. The solution of the initial value problem of the system (1) exists and is unique on $\mathbb{R}_{+}^{1}$.

Proof. To begin with, we show that $x_{1}(t, \varphi)$ exists and is unique on $[0, \tau]$ for any given initial value function $\varphi \in C$. For the convenience of expression, we denote $a(t)=\varphi_{\overline{2}}\left(t-r_{\overline{2}}\right)$ for $t \in[0, \tau]$. Obviously, according to Lemma 2.2 in [16], one can obtain that the solution of the initial value problem of the below system exists and is unique on $[0, \tau]$,

$$
\left\{\begin{array}{l}
y^{\prime}(t)=-F\left(y(t)+c_{\overline{2}} a(t)\right)+G(a(t)) \\
y(0)=\varphi_{1}(0)-c_{\overline{2}} \varphi_{\overline{2}}\left(-r_{\overline{2}}\right)
\end{array}\right.
$$

Denote $\widetilde{y}(t)=x_{1}(t, \varphi)-c_{\overline{2}} \varphi_{\overline{2}}\left(t-r_{\overline{2}}\right)$, then $\widetilde{y}(t)$ satisfies (2); therefore, $x_{1}(t, \varphi)$ exists and is unique on $[0, \tau]$. Using a similar method, one can also prove that $x_{i}(t, \varphi)$ exists and 
is unique on $[0, \tau]$ for $i=2,3, \cdots, n$. Therefore, the mathematical induction can be directly applied to know that Lemma 1 is indeed true.

As usual, we denote $O(\varphi)=\left\{x_{t}(\varphi) \mid \varphi \in C, t \geq 0\right\}, \overline{O(\varphi)}$ is the closure of $O(\varphi)$, and $\omega(\varphi)=\bigcap_{t \geq 0} \overline{O\left(x_{t}(\varphi)\right)}$. If $O(\varphi)$ is bounded, then $\overline{O(\varphi)}$ is compact in $C$, and $\omega(x)$ is nonempty, compact, invariant, and connected.

Next, we give the following lemmas, describing the monotonicity of the system (1).

Lemma 2. If $\varphi \geq_{K} \widehat{\alpha}$ holds for some $\varphi \in C$ and $\alpha \in \mathbb{R}^{1}$, then $x_{t}(\varphi) \geq_{K} \widehat{\alpha}$ holds for all $t \in \mathbb{R}_{+}^{1}$.

Proof. For all $t \in \mathbb{R}_{+}^{1}$ and $i \in \Omega$, we designate $y_{i}(t)=x_{i}(t, \varphi)-c_{\overline{i+1}} x_{\overline{i+1}}\left(t-r_{\overline{i+1}}, \varphi\right)$. Then for all $t \in \mathbb{R}_{+}^{1}$ and $i \in \Omega$, we assert that $y_{i}(t) \geq\left(1-c_{\overline{i+1}}\right) \alpha$. In fact, assuming the opposite is true, one can choose $i_{0} \in \Omega$ and $t_{0} \in(0, \tau]$, such that

$$
y_{i_{0}}\left(t_{0}\right)<\left(1-c_{\overline{i_{0}+1}}\right) \alpha, \text { and } y_{i_{0}}^{\prime}\left(t_{0}\right)<0 \text {. }
$$

Therefore, we have

$$
\begin{aligned}
& x_{i_{0}}\left(t_{0}, \varphi\right) \\
< & \left(1-c_{\overline{i_{0}+1}}\right) \alpha+c c_{\overline{i_{0}+1}} x_{\overline{i_{0}+1}}\left(t_{0}-r_{\overline{i_{0}+1}}, \varphi\right) \\
\leq & x_{\overline{i_{0}+1}}\left(t_{0}-r_{\overline{i_{0}+1}}, \varphi\right) .
\end{aligned}
$$

By systems (1) and the monotonicity of function $F$, we have the following inequality

$$
\begin{aligned}
& y_{i_{0}}^{\prime}\left(t_{0}\right) \\
= & -F\left(x_{i_{0}}\left(t_{0}, \varphi\right)\right)+G\left(x_{\overline{i_{0}+1}}\left(t_{0}-r_{\overline{i_{0}+1}}, \varphi\right)\right) \\
\geq & -F\left(x_{i_{0}}\left(t_{0}, \varphi\right)\right)+F\left(x_{\overline{i_{0}+1}}\left(t_{0}-r_{\overline{i_{0}+1}}, \varphi\right)\right) \\
> & 0,
\end{aligned}
$$

which contradicts the assumption $y_{i_{0}}^{\prime}\left(t_{0}\right)<0$, and proves the assertion.

Applying the assertion and Remark 2 (i), we have $x_{t}(\varphi) \geq_{K} \widehat{\alpha}$ for all $t \in[0, \tau]$. Then Lemma 2 follows by the method of mathematical induction.

Lemma 3. Under the hypotheses of Lemma 2, if $\varphi_{i}(0)-c_{\overline{i+1}} \varphi_{\overline{i+1}}\left(-r_{\overline{i+1}}\right)>\left(1-c_{\overline{i+1}}\right) \alpha$ for some $i \in \Omega$, then $x_{\overline{i-1}}\left(r_{i}, \varphi\right)-c_{i} x_{i}(0, \varphi)>\left(1-c_{i}\right) \alpha$.

Proof. Otherwise, $x_{\overline{i-1}}\left(r_{i}, \varphi\right)-c_{i} x_{i}(0, \varphi)=\left(1-c_{i}\right) \alpha$. Applying Lemma 2, we get $\left(x_{\overline{i-1}}\right.$ $\left.(t, \varphi)-c_{i} x_{i}\left(t-r_{i}, \varphi\right)\right)\left.^{\prime}\right|_{t=r_{i}}=0$.

It follows from (1) that $F\left(x_{\overline{i-1}}\left(r_{i}, \varphi\right)\right)=G\left(x_{i}(0, \varphi)\right) \geq F\left(x_{i}(0, \varphi)\right)$ and hence $x_{\overline{i-1}}\left(r_{i}, \varphi\right)$ $\geq x_{i}(0, \varphi)=\varphi_{i}(0)>\alpha$.

However, by Remark 1, we then obtain $x_{i-1}\left(r_{i}, \varphi\right)<x_{i}(0, \varphi)$, which yields a contradiction.

Lemma 4. Assume $O(\varphi)$ is bounded for some $\varphi \in C$. Define $\alpha^{*}=\sup \left\{\alpha \in \mathbb{R}^{1}, \widehat{\alpha} \leq_{K} \omega(\varphi)\right\}$. Then we have the below statements:

- (i) For $i \in \Omega$, if $\psi \in \omega(\varphi)$, then one can choose $s^{*} \in[0,|r|]$, such that

$$
x_{i}\left(s^{*}, \psi\right)-c_{\overline{i+1}} x_{\overline{i+1}}\left(s^{*}-r_{\overline{i+1}}, \psi\right)=\left(1-c_{\overline{i+1}}\right) \alpha^{*} .
$$

- (ii) For $i \in \Omega$, if $\psi \in \omega(\varphi)$, then one can choose $s^{*} \in[0,|r|]$ such that

$$
x_{i}\left(s^{*}+k|r|+\sum_{j=i+1}^{n} r_{j}, \psi\right)-c_{\overline{i+1}} x_{i+1}\left(s^{*}+k|r|+\sum_{j=i+2}^{n} r_{j}, \psi\right)=\left(1-c_{\overline{i+1}}\right) \alpha^{*},
$$


where $k \geq 0$ is an integer.

Proof. (i) By the symmetry of (1), we only need to consider the case $i=n$. Suppose the result is not true, one can choose $\psi \in \omega(\varphi)$ such that

$$
x_{n}(t, \psi)-c_{1} x_{1}\left(t-r_{1}, \psi\right)>\left(1-c_{1}\right) \alpha^{*}, \quad t \in[0,|r|] .
$$

Iterating Lemma $3 n-1$ times, we therefore have

$$
x_{i}(t, \psi)-c_{\overline{i+1}} x_{\overline{i+1}}\left(t-r_{\overline{i+1}}, \psi\right)>\left(1-c_{\overline{i+1}}\right) \alpha^{*},
$$

where $t \in\left[\sum_{j=i+1}^{n} r_{j},|r|\right], \quad i \in\{1,2, \cdots, n-1\}$.

It follows from Remark 2 that we have $x_{|r|}(\psi) \gg_{K} \widehat{\alpha^{*}}$. Applying Lemma 2 and combining the concept of $\omega(\varphi)$, we can choose $\beta^{*}>\alpha^{*}$ such that $\omega(\varphi) \geq_{K} \widehat{\beta^{*}} \gg_{K} \widehat{\alpha^{*}}$. Note that this conflicts with the definition of $\alpha^{*}$. This completes the proof of the statement (i).

(ii) For all $i \in \Omega$ and $k \geq 0$, we denote

$A_{i}^{k}=\left\{s \in[0,|r|]: x_{i}\left(s+k|r|+\sum_{j=i+1}^{n} r_{j}, \psi\right)-c_{\overline{i+1}} x_{i+1}\left(s+k|r|+\sum_{j=i+2}^{n} r_{j}, \psi\right)=\left(1-c_{\overline{i+1}}\right) \alpha^{*}\right\}$.

Then $A_{i}^{k} \neq \phi$ follows from (i).

For $i=1,2, \cdots, n-1, k \geq 0$, applying Lemma 3, we obtain $A_{i}^{k} \subseteq A_{i+1}^{k}$ and $A_{n}^{k+1} \subseteq A_{1}^{k}$. Consequently, $\bigcap_{k=0}^{\infty} \bigcap_{i=1}^{n} A_{i}^{k} \neq \phi$. We choose $s^{*} \in \bigcap_{k=0}^{\infty} \bigcap_{i=1}^{n} A_{i}^{k}$ as required.

\section{Main Results}

Based on the relevant preparations in the previous section, we can now present and demonstrate the main results of this article in this section.

Theorem 1. If $O(\varphi)$ is bounded for some $\varphi \in C$, and $G(x) \geq F(x)$ for all $x \in \mathbb{R}^{1}$, then one can choose $\alpha^{*} \in \mathbb{R}^{1}$, such that $\omega(\varphi)=\left\{\widehat{\alpha^{*}}\right\}$.

Proof. Define $\alpha^{*}=\sup \left\{\alpha \in \mathbb{R}^{1}: \widehat{\alpha} \leq_{K} \omega(\varphi)\right\}$. We will prove that $\omega(\varphi)=\left\{\widehat{\alpha^{*}}\right\}$; otherwise, we have $\omega(\varphi) \backslash\left\{\widehat{\alpha^{*}}\right\} \neq \phi$.

By combining Lemma 4 with the invariance of $\omega(\varphi)$, one can choose $\psi \in \omega(\varphi) \backslash\left\{\widehat{\alpha^{*}}\right\}$, such that

$$
x_{i}\left(k|r|+\sum_{j=i+1}^{n} r_{i}, \psi\right)-c_{\overline{i+1}} x_{i+1}\left(k|r|+\sum_{j=i+2}^{n} r_{j}, \psi\right)=\left(1-c_{\overline{i+1}}\right) \alpha^{*}
$$

for $i \in \Omega$ and $k \geq 0$.

Integrating (1), we have

$$
\begin{gathered}
\int_{k|r|+s+\sum_{j=i+1}^{n} r_{j}}^{(k+1)|r|+\sum_{j=i+1}^{n} r_{j}}\left(x_{i}(t, \psi)-c_{\overline{i+1}} x_{\overline{i+1}}\left(t-r_{\overline{i+1}}, \psi\right)\right)^{\prime} d t \\
=\quad \int_{k|r|+s+\sum_{j=i+1}^{n} r_{j}}^{(k+1)|r|+\sum_{j=i+1}^{n} r_{j}}-F\left(x_{i}(t, \psi)\right)+G\left(x_{\overline{i+1}}\left(t-r_{\overline{i+1}}, \psi\right)\right) d t .
\end{gathered}
$$


Let

$$
\begin{aligned}
& H_{i}^{k}(s)=\int_{k|r|+s+\sum_{j=i+1}^{n} r_{j}}^{(k+1)|r|+\sum_{j=i+1}^{n} r_{j}} G\left(x_{i}(t, \psi)\right) d t, \\
& h_{i}^{k}(s)=\int_{k|r|+s+\sum_{j=i+1}^{n} r_{j}^{n}}^{(k+1)|r|+\sum_{j=i+1}^{n} r_{j}} F\left(x_{i}(t, \psi)\right) d t,
\end{aligned}
$$

where $s \in[0,|r|], k \geq 0$ and $i \in \Omega$. It follows from (3) and (4), we have

$$
\begin{aligned}
& \left(1-c_{i+1}\right) \alpha-\left(x_{i}\left(k|r|+s+\sum_{j=i+1}^{n} r_{j}, \psi\right)\right. \\
& \left.-c_{i+1} x_{i+1}\left(k|r|+s+\sum_{j=i+1}^{n} r_{j}, \psi\right)\right) \\
= & -h_{i}^{k}(s)+H_{i+1}^{k}(s),
\end{aligned}
$$

with $k \geq 0, i=1,2, \cdots, n-1$, and

$$
\begin{aligned}
& \left(1-c_{1}\right) \alpha^{*}-\left(x_{n}(k|r|+s, \psi)-c_{1} x_{1}\left(k|r|-r_{1}, \psi\right)\right) \\
= & -h_{n}^{k}(s)+H_{1}^{k-1}(s), k \geq 1 .
\end{aligned}
$$

Let $\xi_{i}^{k}(s)=x_{i}\left(k|r|+s+\sum_{j=i+1}^{n} r_{j}, \psi\right)-\alpha^{*}$ for all $i \in \Omega, k \geq 0$ and $s \in[0,|r|]$. Then for $k \geq 1$ and $s \in[0,|r|]$, we have

$$
\left\{\begin{array}{rcl}
-\xi_{1}^{k}(s)+c_{2} \xi_{2}^{k}(s) & \geq-h_{1}^{k}(s)+h_{2}^{k}(s), \\
-\xi_{2}^{k}(s)+c_{3} \xi_{3}^{k}(s) & \geq-h_{2}^{k}(s)+h_{3}^{k}(s), \\
& \cdots \cdots & \\
-\xi_{n-1}^{k}(s)+c_{n} \xi_{n}^{k}(s) & \geq-h_{n-1}^{k}(s)+h_{n}^{k}(s), \\
-\xi_{n}^{k}(s)+c_{1} \xi_{1}^{k-1}(s) & \geq-h_{n}^{k}(s)+h_{1}^{k-1}(s) .
\end{array}\right.
$$

Applying (5), we obtain

$$
\xi_{1}^{k}(s)-c_{1} \xi_{1}^{k-1}(s)+\sum_{i=2}^{n}\left(1-c_{i}\right) \xi_{i}^{k}(s) \leq h_{1}^{k}(s)-h_{1}^{k-1}(s)
$$

Consequently, for any positive integers $\mathrm{N}$, by (6), we have

$$
\sum_{k=1}^{N} \sum_{i=1}^{n}\left(1-c_{i}\right) \xi_{i}^{k}(s) \leq h_{1}^{N}(s)-h_{1}^{0}(s)+c_{1} \xi_{1}^{0}(s)-c_{1} \xi_{1}^{N}(s) .
$$
that

Making full use of the compactness property of $\omega(\varphi)$, one can choose $M>0$, such

$$
\left|h_{i}^{k}(s)\right| \leq M \quad \text { and } \quad\left|\xi_{i}^{k}(s)\right| \leq M .
$$

Hence, $\sum_{k=1}^{N}\left(1-c_{i}\right) \xi_{i}^{k}(s) \leq 4 M$ holds for $N \geq 1, i \in \Omega$ and $s \in[0,|r|]$. Thus, it is easy to see that for any $i \in \Omega, \xi_{i}^{k}(\cdot)$ uniformly convergent to 0 in $[0,|r|]$ as $k \rightarrow \infty$. Therefore,

$$
\lim _{k \rightarrow \infty} h_{i}^{k}(0)=F\left(\alpha^{*}\right)|r|
$$

On the other hand, applying (5) with $s=0$, we obtain $h_{i+1}^{k}(0) \leq h_{i}^{k}(0)$ for all $i=1,2, \cdots, n-1$ and $h_{1}^{k-1}(0) \leq h_{n}^{k}(0)$, so that $h_{i}^{k}(0) \geq h_{i}^{0}(0)$ for all $i \in \Omega$ and $k \geq 0$. Since $\psi \in \omega(\varphi) \backslash\left\{\widehat{\alpha^{*}}\right\}$ and $F$ is strictly increasing, there exists $i_{0} \in\{1,2, \cdots, n\}$, such that $h_{i_{0}}^{0}(0)>F\left(\alpha^{*}\right)|r|$. 
Thus $h_{i_{0}}^{k}(0) \geq h_{i_{0}}^{0}(0)>F\left(\alpha^{*}\right)|r|$, which contradicts with $\lim _{k \rightarrow \infty} h_{i_{0}}^{k}=F\left(\alpha^{*}\right)|r|$. Therefore, $\omega(\varphi)=\left\{\widehat{\alpha^{*}}\right\}$. This finishes the proof of Theorem 1 .

Theorem 2. If $O(\varphi)$ is bounded for some $\varphi \in C$, and $G(x) \leq F(x)$ for all $x \in \mathbb{R}^{1}$, then one can choose $\alpha^{*} \in \mathbb{R}^{1}$, such that $\omega(\varphi)=\left\{\hat{\alpha}^{*}\right\}$.

Proof. To begin with, for all $x \in \mathbb{R}^{1}$, we define $f(x), y(t), g(x)$ as below

$$
f(x)=-F(-x), \quad y(t)=-x(t, \varphi), \text { and } g(x)=-G(-x) .
$$

Then it is obvious that $y(t)$ satisfies system (1) by replacing $F, G$ with $f, g$, respectively. Consequently, directly using the method similar to Theorem 1 , we can easily prove that Theorem 2 is true.

Remark 3. For all $x \in \mathbb{R}^{1}$, if $G(x) \leq F(x)$ and $\varphi \leq_{K} \widehat{\alpha}$ holds for some $\varphi \in C$ and $\alpha \in \mathbb{R}^{1}$, then directly employing the method similar to Lemma 2, one can easily establish the statement that $x_{t}(\varphi) \leq_{K} \widehat{\alpha}$ for all $t \in \mathbb{R}_{+}^{1}$.

Thus, when $G(x) \leq F(x)$, applying Lemma 2 and the above statement, it is easy to reveal that every solution of (1) is bounded. Furthermore, based on the above two theorems, we can directly establish Corollary 1 as below.

Corollary 1. For system (1), if $G(x) \equiv F(x)$ is always true, then every solution converges to a constant vector as t tends to infinity.

Remark 4. Clearly, it follows Corollary 1 that the high-dimensional Haddock conjecture is true. Our main results in this paper improve and generalize the corresponding results obtained in [16,17,19-22]. In particular, our proofs are quite different from those of [13-17,19-22]. Furthermore, it should be noted that system (1) and its generalizations have been used by extensive recent literature studies [23-30] to simulate the dynamical behaviors of delayed neural network models. However, these literature studies treat the global Lipschz condition as the basic additional assumption to the functions $F$ and $G$, which indicates that the results of this present paper are completely different from the corresponding conclusions in the above literature.

Remark 5. It is worth noting that the critical case of system (1) with $c_{i} \equiv 1$ for $i \in \Omega$ has been investigated in the literature [31] under the relatively strict additional conditions $\left(\mathbf{S}_{+}\right)$or $\left(\mathbf{S}_{-}\right)$. The differences between our paper and the literature [31] mainly lie in the following two aspects. On the one hand, if one choose $G(x) \equiv F(x)=x^{\frac{1}{3}}$ designed in the Haddock conjecture, simple verification can guide that the function $F$ and $G$ do not meet the requirements of conditions $\left(\mathbf{S}_{+}\right)$or $\left(\mathbf{S}_{-}\right)$. Obviously, the investigation in our paper removes the assumptions $\left.\mathbf{( S}_{+}\right)$and $\left(\mathbf{S}_{-}\right)$and overcomes its shortcomings. On the other hand, the main results are completely different; the $\omega$-limit set in literature [31] may be periodic solutions, which cannot ensure that each solution converges to a constant vector, but our conclusions clearly point out that each solution must converge to a constant vector. Therefore, on a broader level, our conclusion can be regarded as a high-dimensional version of the famous Haddock conjecture. Since the assumption of a monotonic function in biology is rather vague, and most stochastic dynamics do not always tend towards a strict monotonicity, it would be interesting to further study the deviations of the conjecture in such typical cases. This is our future research direction.

Author Contributions: Formal analysis, C.H. and W.W.; methodology, L.L. and X.Y.; writingoriginal draft preparation, C.H. and W.W.; writing—review and editing, C.H., W.W., L.L. and X.Y. All authors have read and agreed to the published version of the manuscript.

Funding: This work was supported by the National Natural Science Foundation of China (No. 11971076). 
Institutional Review Board Statement: Not applicable.

Informed Consent Statement: Not applicable.

Data Availability Statement: Not applicable.

Acknowledgments: The authors would like to thank the reviewers and the editor for the helpful suggestions and comments, which led to improvements of our original paper.

Conflicts of Interest: We confirm that we have no conflict of interest.

\author{
Abbreviations \\ The following abbreviations are used in this manuscript: \\ NFDE Neutral functional differential equation \\ Iff If and only if
}

\title{
References
}

1. Wu, J.; Zhang, X. Transmission Dynamics of Tick-Borne Diseases with Co-Feeding, Developmental and Behavioural Diapause; Lecture Notes on Mathematical Modelling in the Life Sciences; Springer: Cham, Switzerland, 2020.

2. Zhu, Q. Stabilization of stochastic nonlinear delay systems with exogenous disturbances and the event-triggered feedback control. IEEE Trans. Autom. Control 2019, 64, 3764-3771. [CrossRef]

3. Zhu, Q. Stability analysis for a class of stochastic delay nonlinear systems driven by G-Brownian motion. Syst. Control Lett. 2020, 140, 104699. [CrossRef]

4. Ding, K.; Zhu, Q. Extended dissipative anti-disturbance control for delayed switched singular semi-Markovian jump systems with multi-disturbance via disturbance observer. Automatica 2021, 18, 109556. [CrossRef]

5. Hale, J.K.; Lunel, S.M.V. Introduction to Functional Differential Equations; Springer: New York, NY, USA, 1993.

6. Tan, Y. Dynamics analysis of mackey-glass model with two variable delays. Math. Biosci. Eng. 2020, 17, 4513-4526. [CrossRef] [PubMed]

7. Huang, C.; Tan, Y. Global behavior of a reaction-diffusion model with time delay and Dirichlet condition. J. Differ. Equ. 2021, 271, 186-215. [CrossRef]

8. Long, X. Novel stability criteria on a patch structure Nicholson's blowflies model with multiple pairs of time-varying delays. AIMS Math. 2020, 5, 7387-7401. [CrossRef]

9. Tan, Y.; Huang, C.; Sun, B.; Wang, T. Dynamics of a class of delayed reaction-diffusion systems with Neumann boundary condition. J. Math. Anal. Appl. 2018, 458, 1115-1130. [CrossRef]

10. Hu, H.; Yi, T.; Zou, X. On spatial-temporal dynamics of a fisher-kpp equation with a shifting environment. Proc. Am. Math. Soc. 2020, 148, 213-221. [CrossRef]

11. Hu, H.; Zou, X. Existence of an extinction wave in the fisher equation with a shifting habitat. Proc. Am. Math. Soc. 2017, 145, 4763-4771. [CrossRef]

12. Long, X.; Gong, S. New results on stability of Nicholson's blowflies equation with multiple pairs of time-varying delays. Appl. Math. Lett. 2020, 2020, 106027. [CrossRef]

13. Haddock, J. Functional differential equations for which each constant function is a solution: A narrative. In Proceedings International Conference on Nonlinear Oscillations; Farkas, M., Kertesz, V., Stepan, G., Eds.; Janos Bolyai Math. Soc.: Budapest, Hungary, 1987; pp. 86-93.

14. Yi, T. A generalization of the Haddock conjecture and its proof. Nonlinear Anal. Real World Appl. 2008, 9, 1112-1118. [CrossRef]

15. Xiao, S. A New Generalization of the Haddock Conjecture. Math. Model. Anal. 2017, 22, 634-642. [CrossRef]

16. Xiong, W.; Liu, B. Asymptotic behavior of bounded solutions for a class of systems of neutral functional differential equations. J. Math. Anal. Appl. 2006, 313, 754-760. [CrossRef]

17. Xiong, W.; Wang, L. Corrigendum to "Asymptotic behavior of bounded solutions for a system of neutral functional differential equations" [J. Math. Anal. Appl. 313 (2006) 754-760]. J. Math. Anal. Appl. 2009, 350, 423. [CrossRef]

18. Yang, H. Weighted pseudo almost periodicity on neutral type CNNs involving multi-proportional delays and D operator. AIMS Math. 2021, 6, 1865-1879. [CrossRef]

19. Meng, J. Asymptotic behavior of bounded solutions for a system of neutral functional differential equations. Nonlinear Anal. Real World Appl. 2010, 11, 1634-1639. [CrossRef]

20. Gao, H.; Long, H. Dynamic behavior for a system of neutral functional differential equations. Comput. Math. Appl. 2009, 58, 2046-2050. [CrossRef]

21. Xu, M.; Yuan, Z.; Wang, W.; Long, H. $\omega$ limit sets of solutions for a class of neutral functional differential equations. Nonlinear Anal. Real World Appl. 2010, 11, 2345-2349. [CrossRef]

22. Kong, F.; Luo, Z. Asymptotic behavior of bounded solutions to a system of neutral functional differential equations in critical case. Appl. Math. Lett. 2018, 81, 44-49. [CrossRef] 
23. Xiao, S. Global exponential convergence of HCNNs with neutral type proportional delays and D operator. Neural Process. Lett. 2019, 49, 347-356. [CrossRef]

24. Xu, C.; Li, P.; Xiao, Q.; Yuan, S. New results on competition and cooperation model of two enterprises with multiple delays and feedback controls. Bound. Value Probl. 2019, 2019, 36. [CrossRef]

25. Wang, J.; Chen, X.; Huang, L. The number and stability of limit cycles for planar piecewise linear systems of node-saddle type. J. Math. Anal. Appl. 2019, 469, 405-427. [CrossRef]

26. Duan, L.; Huang, L.; Guo, Z.; Fang, X. Periodic attractor for reaction-diffusion high-order Hopfield neural networks with time-varying delays. Comput. Math. Appl. 2017, 73, 233-245. [CrossRef]

27. Cao, Q.; Long, X. New convergence on inertial neural networks with time-varying delays and continuously distributed delays. AIMS Math. 2020, 5, 5955-5968. [CrossRef]

28. Yang, C.; Huang, L.; Li, F. Exponential synchronization control of discontinuous nonautonomous networks and autonomous coupled networks. Complexity 2018, 2018, 6164786. [CrossRef]

29. Wang, H.; Liu, L.; Tan, Y. Dynamics of Third-Order Nonlinear Neutral Equations. Discrete Dyn. Nat. Soc. 2014, $2014,1-4$. [CrossRef]

30. Wang, J.; Huang, L. Limit cycles bifurcated from a focus-fold singularity in general piecewise smooth planar systems. J. Differ. Equ. 2021, 304, 491-519. [CrossRef]

31. Zhang, X.; Hu, H. Convergence in a system of critical neutral functional differential equations. Appl. Math. Lett. 2020, 107, 106385. [CrossRef] 